\title{
Analysis of Novel Metaphor From Blending Relevance Theory
}

\author{
XIE Ying-mei \\ Jiangsu Shipping College, Nantong, China
}

\begin{abstract}
Novel metaphor is different from the conventional metaphor. The distinction between novel metaphor and conventional metaphor is not clear-cut but in a degree of metaphoricity. In the situation, when novel metaphors are widely accepted and generally used in daily life, their metaphorical meaning are neglected by people, the metaphorical characters decrease and they become conventional metaphors. The tentative model Blending Relevance Theory (BRT) is an integrated framework of the two theories: Conceptual Blending Theory (CBT) and Relevance Theory (RT), seeking the optimal relevance from the two input spaces.
\end{abstract}

Keywords: novel metaphor, CBT, RT, BRT

\section{Novel Metaphor}

Novel metaphor in sitcom, as the subject matter for the present research, is different from the conventional metaphor. In Metaphor We Live By, Lakoff and Johnson (2003) emphasized that conventional metaphor is such a kind of metaphor that "structures(s) the ordinary conceptual system of our culture, which is reflected in our everyday language" (p. 140), while novel metaphor is "a metaphor not used to structure part of our normal conceptual system but as a new way of thinking about something..." (p. 54).

\section{Different Statements of Novel Metaphor}

Liu (1999) stated all new metaphorical utterances derive from the existing conceptual metaphors in the long memory and the creativity and novelty seemed to be written off in Lakoff's theory. However, existing conceptual metaphors in the long memory is still unknown. Inspire of this, culture factors and context and are very essential to the generation of novel metaphors.

Lakoff and Johnson (2003) also stated that "if a new metaphor enters the conceptual system that we base our actions on, it will alter that conceptual system and the perceptions and actions that the system gives rise to" (p. 146). Therefore, "novel metaphor can be treated as similarity-creating metaphor, which can generate new perceptions, explanations and inventions" (Wang, 2011, p. 26). That is, novel metaphor is a kind of recategorization or reconceptulization. Different from conventional metaphor, novel metaphor is more creative and imaginative with new meaning. They make people recognize things from a different aspect and reconceptualize them accordingly.

\section{Distinction Between Novel Metaphor and Conventional Metaphor}

The distinction between novel metaphor and conventional metaphor is not clear-cut but in a degree of metaphoricity. In the situation, when novel metaphors are widely accepted and generally used in the daily life, their metaphorical meaning are neglected by people, the metaphorical characters decrease and they become 
conventional metaphors. Moreover, different people have different views on novel metaphors and conventional metaphors. A conventional metaphor may be understood as a novel metaphor to one person, or a novel metaphor may be explained as a conventional metaphor to another person. It relies on the cognitive background.

When it comes to novel metaphor, $\mathrm{Hu}$ Zhuanglin (2004) distinguished the conventional from unconventional metaphor, arguing that the latter includes literary metaphor or to be exact and poetic metaphor, but it can also be used beyond the literary works (pp. 154-57).

Shu Dingfang (2000) drew a distinction between the similarity-based and similarity-creating metaphor. In terms of the similarity-based metaphor, there exists some kind of similarity between the topic and vehicle, which people have known before they use such metaphor whereas the similarity-creating metaphor is such metaphor that before it is used, people have no idea about the similarity between the topic and vehicle (p. 58).

According to Halliday's register theory, Goatly (1997, as cited in Shu, 2000) made an explanation that the frequencies of novel metaphors in different registers are various (see Table 1).

Table 1

Frequencies of Novel Metaphors in Different Registers

\begin{tabular}{lc}
\hline Register & Novel metaphor (\%) \\
\hline Verbal communication & 10 \\
News report & 4 \\
Science books & 18 \\
Magazine and Ads. & 22 \\
Modern novels & 28 \\
Modern poetry & 56 \\
\hline
\end{tabular}

Note. Goatly, 1997, p. 332, as cited in Shu, 2000, p. 256.

\section{A Tentative Analytic Model: Integrating CBT and RT}

An integrated analysis of Conceptual Blending Theory (CBT) and Relevance Theory (RT) was first conducted by Jiang and Ma (2003). The feasibility for such an analysis based on the two theories rests upon the presumption that they have common ground.

\section{Similarities of the Two Theories}

Jiang and Ma (2003) proposed that the basement of integrating CBT and RT is the similar language principles. Econ in common.omical principle of encoding and the idea of on-line blending are the two aspects that they have

Economical principle of encoding. In Gestalt principle of perception, our mind understands wholes out of incomplete elements. Fauconnier (1994) suggested that the same language form can be properly used in different situations, because language form is very simple. The concept structure intended by the utterance is not absolutely clearly represented by its semantic structure. The communicator's activity is governed by the principle of minimal encoding effort, which indicates that with minimal encoding efforts, a large amount of information will get across to our addressee.

On-line blending. The CBT and RT focus on on-line inferential process, emphasizing the role of working memory in information processing. In CBT, semantics depends on on-line association, which means the 
capability of conceptual blending and cross-domain projection. The variable association results in the uncertainty of meaning construction. Cognitive activity happens at different time points, thus marked by time. It is plausible to confirm that there is constant blending process in our conceptual system.

RT believes that discourse comprehension is a kind of process involving both decoding and inferential. In order to reach conclusion, the process includes activating contextual assumptions, recognizing encoded information, establishing a matching relation between contextual assumptions and encoded information, making use of encoded messages and contextual assumptions for inference.

\section{Grounds for Integrating CBT and RT}

Studies on the CBT and RT have provided some grounds for integrating the two theories.

The followings are the reasons for integrating the two theories to explain metaphors:

Firstly, CBT states that language helps to put forward the cognitive constructions by means of clues and cues which are very partial, but contextually efficient. In RT, utterance is propositional and encoded economically to be cost-efficient, while the conceptual structures are much more than what are directly expressed.

Secondly, CBT focuses on local and dynamic meaning construction, which is inherited from Mental Space Theory. In RT, factors, such as recognizing encoded information, activating contextual assumptions, establishing a matching relation between contextual assumptions and encoded messages, should be involved in utterance understandings.

Thirdly, CBT and RT are complementary. In CBT, the blend is a third space yielded by the operation on two input mental spaces. In the conceptual integration network, input mental spaces are retrievable in the blending model. However, when analyzing the blending process, theorists retrieve the input mental spaces by the emergent structure which is expected by the theorists.

This thesis proposes a blending-relevance model in the light of RT and CBT with the aim to explore the cognitive mechanism of novel metaphor.

\section{Analysis of Novel Metaphor from BRT, a Tentative Analytic Model}

The tentative model BRT is an integrated framework of the two theories, CBT and RT, seeking the optimal relevance from the two input spaces. RT emphasizes that comprehension of metaphors is a dynamic process in which the hearer searches for the optimal relevance and obtains contextual effect under proper cognitive context. While CBT exploits the on-line psychological cognitive mechanism which is hidden behind metaphors, and emphasizes that metaphors are the blending of two or more input spaces, the most important point lies in the blending of the semantic elements from each space, then resulting in the emergent structure.

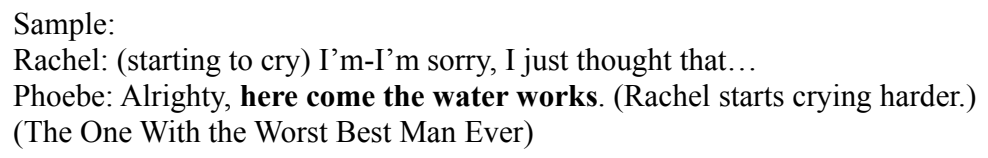

The conversation of sample happened in the situation when Phoebe talked about the period of his life wandering in the street. Rachel asked about some details and then felt very sad for the hardship that Phoebe experienced. After listening, Rachel began to cry. Seeing it, Phoebe responded "Here come the water works". Therefore, in Sample 51, Input Space I is Rachel starting to cry; Input Space II is water falling as the rain-wash. The Genetic Space of the mapping is the common structure of the two events, which together with the content 
of the two Input Spaces, are all projected onto the Blended Space. Through the cognitive process which involves the composition, completion, or elaboration, the metaphorical meaning that Phoebe is suggesting Rachel's crying is manifested.

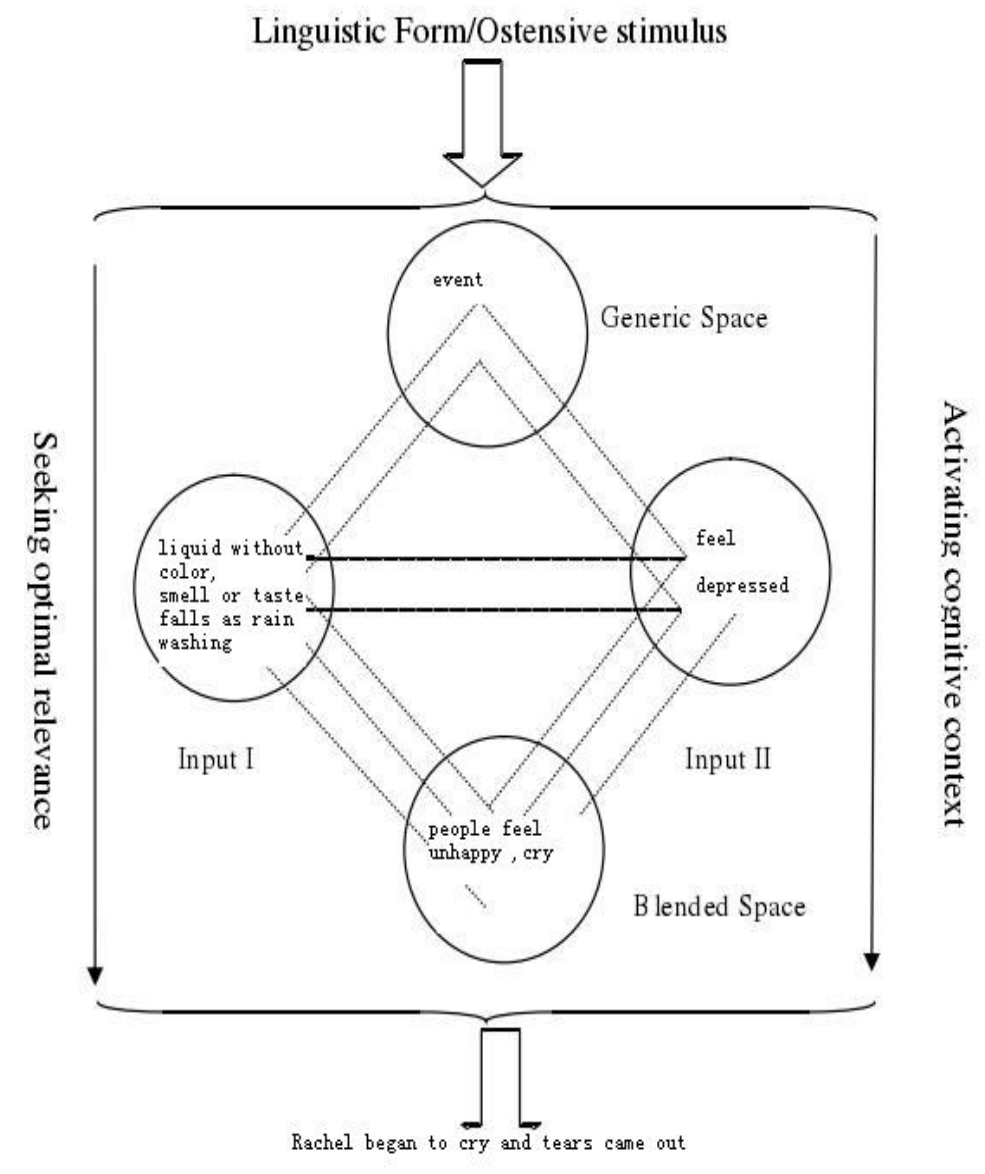

Figure 1. A BRT representation of "Here come the water works".

From the perspective of RT, the hearer may fail when he/she develops the maximal relevance through the word "water", then he/she has to achieve the optimal relevance by associating "water works" with "starting to crying". Clearly, the whole process is a kind of ostensive-inferential communication; the hearer obtains the contextual effects through processing efforts. That is, the resulting product of "crying" is tear, which is a kind of colorless and odorless liquid, much similar to water.

Therefore, the optimal relevance of those two sentences is that tears from Rachel just like the water, and Rachel's crying just like the water works. So, the whole cognitive process of it can be presented in Figure 1.

\section{References}

Coulson, S., \& Oakly, T. (2000). Blending basics. Cognitive Linguistic, 11(3-4), 175-196.

Coulson, S., \& Oakley, T. (2005). Blending and coded meaning: Literal and figurative meaning in cognitive semantics. Journal of Pragmatics, 37(10), 1510-1536.

Fauconnier, G. (1985/1994). Mental space. New York: Cambridge University Press.

Fauconnier, G. (1994). Mental spaces: Aspects of meaning construction in natural language. Cambridge: Cambridge University Press.

Fauconnier, G. (1997). Mappings thought and language (pp. 149-155). Cambridge: Cambridge University Press. 
Hu, Z. L. (2004). Cognitive metaphor. Beijing: Peking University press.

Jiang, Y., \& Ma, L. (2003). An integrative study of SB and RT. Journal of Foreign Languages, (1).

Lakoff, G., \& Johnson, M. (2003). Metaphors we live by (2nd ed.). London: The University of Chicago Press.

Lakoff, G., \& Turner, M. (1980). Metaphors we live by. Chicago: University of Chicago Press.

Shu, D. F. (2000). A study of metaphor. Shanghai: Shanghai Foreign Language Publishing House Education. Sperber, D., \& Wilson, D. (1986). Relevance communication and cognition. London: Oxford Blackwell. 